\title{
Percutaneous left atrial appendage closure for managing thromboembolic risk in atrial fibrillation
}

\author{
Faizan Khan MSc, F. Daniel Ramirez MD, Benjamin Hibbert MD PhD
}

Cite as: CMAJ 2018 October 15;190:E1227-30. doi: 10.1503/cmaj.180470

A trial fibrillation, the most common arrhythmia encountered in clinical practice, is a leading cause of morbidity and mortality ${ }^{1}$ with an incidence that increases with age. Patients with atrial fibrillation, particularly older adults, have a substantially increased risk of thromboembolic stroke. Anticoagulation administered orally is highly effective at reducing the risk of stroke in atrial fibrillation; however, many patients who have atrial fibrillation and are at high risk of stroke (more than half in one study) ${ }^{2}$ are not prescribed oral anticoagulants because of absolute or perceived contraindications (Box 1).

Transesophageal echocardiography studies have suggested that as many as $90 \%$ of cardioembolic strokes in patients with nonvalvular atrial fibrillation originate from the left atrial appendage. ${ }^{4}$ Moreover, strokes related to atrial fibrillation tend to be more severe and have a greater propensity for death or permanent disability. ${ }^{4}$ Methods of occluding the left atrial appendage from systemic circulation have been developed, bolstered by the prospect of protecting against embolic events while avoiding the bleeding risk associated with systemic anticoagulation. In this article, we describe percutaneous left atrial appendage closure that may offer an alternative for stroke prevention in selected patients with atrial fibrillation, particularly those in whom oral anticoagulant therapy is contraindicated.

\section{What is percutaneous left atrial appendage closure?}

Percutaneous left atrial appendage closure is a transcatheter procedure whereby a device is implanted into the left atrial appendage to exclude it from the systemic circulation. There are two devices currently approved in Canada for use in patients with atrial fibrillation and a rationale for a nonpharmacologic alternative for reducing thromboembolic risk. The Watchman device (Boston Scientific, Saint Paul, Minnesota) (Figure 1A) is a self-expandable nitinol cage, covered with a permeable polyethylene terephthalate membrane, which uses 10 fixation anchors to stabilize it in the left atrial appendage. The Amplatzer Amulet device (St. Jude Medical, Saint Paul, Minnesota) is made of nitinol mesh and consists of two disks (a smaller proximal disk

\section{KEY POINTS}

- Stroke prevention is among the major goals in managing nonvalvular atrial fibrillation.

- Anticoagulant therapy is highly effective at reducing stroke risk, but more than half of patients with atrial fibrillation do not receive anticoagulation because of relative or absolute contraindications.

- Percutaneous closure of the left atrial appendage has been shown to be non-inferior to warfarin for prevention of stroke, with reductions in death and major bleeding.

- In patients with atrial fibrillation who are not candidates for long-term anticoagulation, this procedure may offer an alternative for stroke prevention.

that anchors in the left atrium, and a larger disk designed to cover the os) connected by a "waist" (Figure 1B).

Data pertaining to comparisons of left atrial appendage closure devices are lacking; the SWISS-APERO (Comparison of Amplatzer Amulet vs Watchman Device in Patients Undergoing Left Atrial Appendage Closure) randomized trial (clinicaltrials.gov, no. NCT03399851), aimed at comparing the safety and efficacy of the Watchman and the Amulet device, is currently underway.

\section{How is it performed?}

Left atrial appendage closure is a transvenous transseptal procedure performed under general anesthesia with transesophageal echocardiography guidance, or under conscious sedation using intracardiac echocardiographic guidance (Figure 2; three videos showing the procedure are available online as Appendices 1-3, at www.cmaj.ca/lookup/suppl/doi:10.1503/cmaj.180470/-/DC1). Once secure in the left atrial appendage, the devices are released and reendothelialization occurs over a one- to two-month period. The procedure usually takes about 45 minutes and most patients can be discharged home the same or next day. In the randomized controlled trials (RCTs) that tested the Watchman device, patients with no contraindications to anticoagulation received 45 days of warfarin therapy, followed by acetylsalicylic acid (ASA) and clopidogrel for up to six months, followed by ASA alone indefinitely. ${ }^{5}$ Among 
Box 1: Proposed contraindications to oral anticoagulation in patients with atrial fibrillation*

- Previous bleeding

- Patient refusal or preference

- High bleeding risk

- Frequent falls or frailty

- Need for dual antiplatelet therapy

- Unable to adhere to or monitor warfarin

- Comorbidities

- Previous intracranial hemorrhage

- Allergy

- Occupational risk

- Pregnancy

*Based on data from The Outcomes Registry for Better Informed Treatment of Atrial Fibrillation (ORBIT AF). ${ }^{3}$ Listed in order of frequency.

patients ineligible for oral anticoagulation, dual antiplatelet therapy postprocedure has been suggested to be a safe alternative.,7 Although robust data are lacking for this practice, antithrombotic or antiplatelet therapy after implantation is often individualized based on the patient's risk profile and medical history.

\section{Who is eligible?}

The evidence supporting the use of percutaneous left atrial appendage closure is strongest in patients with atrial fibrillation and $\mathrm{CHADS}_{2}$ score of 1 or more based on the available RCT data and metaanalyses. ${ }^{5,8}$ However, in Canada, left atrial appendage closure is reserved for patients with atrial fibrillation and a $\mathrm{CHADS}_{2}$ score of 1 or more in whom oral anticoagulant therapy has been deemed to be contraindicated, or for patients with treatment failure while on anticoagulation (Box 1). Patients are screened with transesophageal echocardiography or computed tomography to ensure anatomic suitability.

Absolute contraindications to the procedure include hypersensitivity or allergy to any component of the device, and anatomic contraindications including left atrial appendage size or shape, or anatomic contraindication to performing a transseptal puncture. Left atrial appendage thrombus is a relative contraindication, but experienced operators can perform the left atrial appendage closure procedure in exceptional clinical scenarios. ${ }^{9}$

\section{What is the evidence so far?}

\section{Patients eligible for oral anticoagulation}

Two industry-sponsored RCTs, PROTECT AF (Watchman Left Atrial Appendage System for Embolic Protection in Patients with Atrial Fibrillation; 707 patients) and PREVAIL (Watchman LAA Closure Device in Patients With Atrial Fibrillation Versus Long Term Warfarin Therapy; 407 patients), compared the Watchman device to warfarin therapy in patients eligible for anticoagulation taken orally. ${ }^{5}$ These studies were noninferiority trials, designed to show that the new intervention is not "unacceptably less efficacious" compared with warfarin - the gold standard at the time of the trials. The landmark
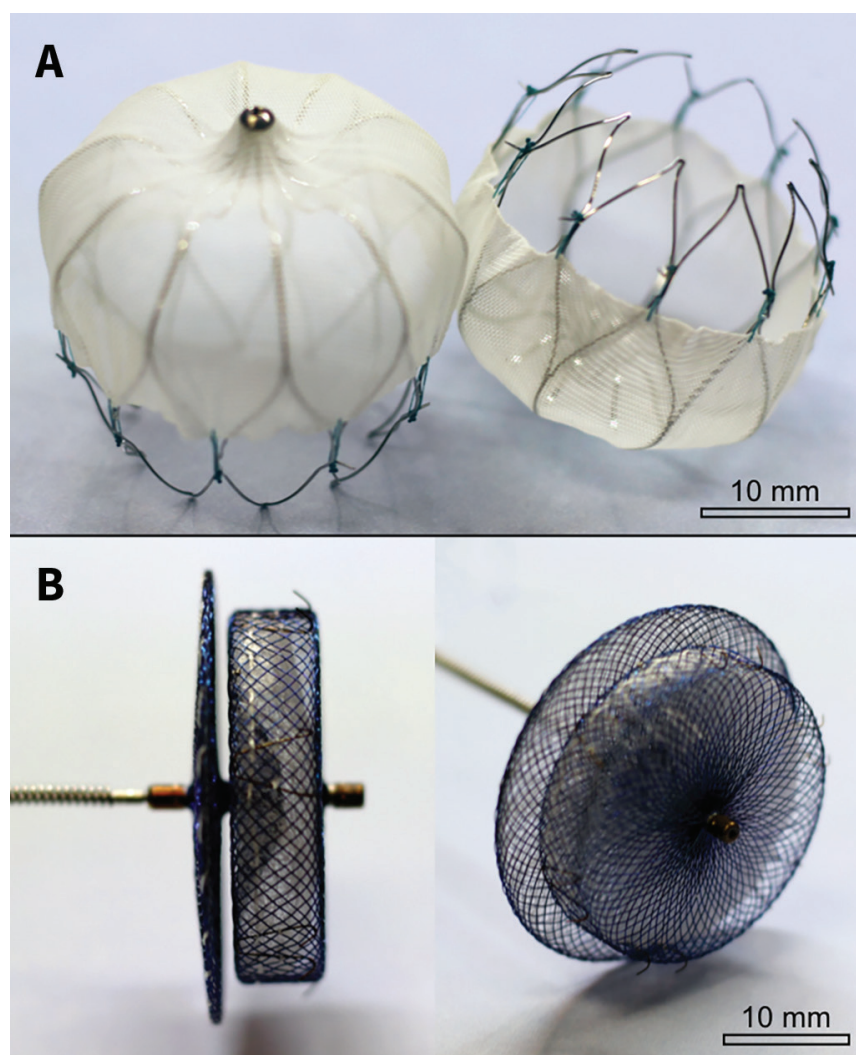

Figure 1: Devices used for percutaneous left atrial appendage closure. (A) Watchman device (Boston Scientific, St. Paul, Minnesota). (B) Amplatzer Amulet device (St. Jude Medical, Saint Paul, Minnesota).

PROTECT AF trial showed that left atrial appendage closure was noninferior to warfarin, reducing the risk of the combined outcome of stroke, systemic embolism and cardiovascular death. Importantly, although there was a reduction in stroke, cardiovascular and all-cause death ${ }^{5}$ compared with warfarin, the rate of ischemic stroke was numerically higher in the left atrial appendage closure arm. The second, smaller PREVAIL trial failed to meet criteria for noninferiority, ${ }^{5}$ although a meta-analysis incorporating all patients from the Watchman RCTs showed noninferiority to warfarin with a significant reduction in all-cause death up to five years. ${ }^{5,8}$ Direct comparisons are lacking, but a recent network meta-analysis comparing left atrial appendage closure to direct oral anticoagulants suggested equivalency in terms of stroke protection. ${ }^{10}$ The ongoing PRAGUE-17 (Interventional Left Atrial Appendage Closure vs. Novel Anticoagulation Agents in High-risk Patients With Atrial Fibrillation; clinicaltrials.gov, no. NCT02426944) randomized trial is likely to provide comparative data relative to direct oral anticoagulants. Based on these findings, the US Food and Drug Administration (FDA) approved Watchman as an alternative to oral anticoagulation for patients with atrial fibrillation in the United States.

\section{Patients with contraindications to oral anticoagulation}

In Canada, transcatheter left atrial appendage closure is approved for patients with a rationale for nonpharmacologic alternative to oral anticoagulation; however, data in this population are less robust. The single-arm, multicentre observational ASAP (ASA Plavix 


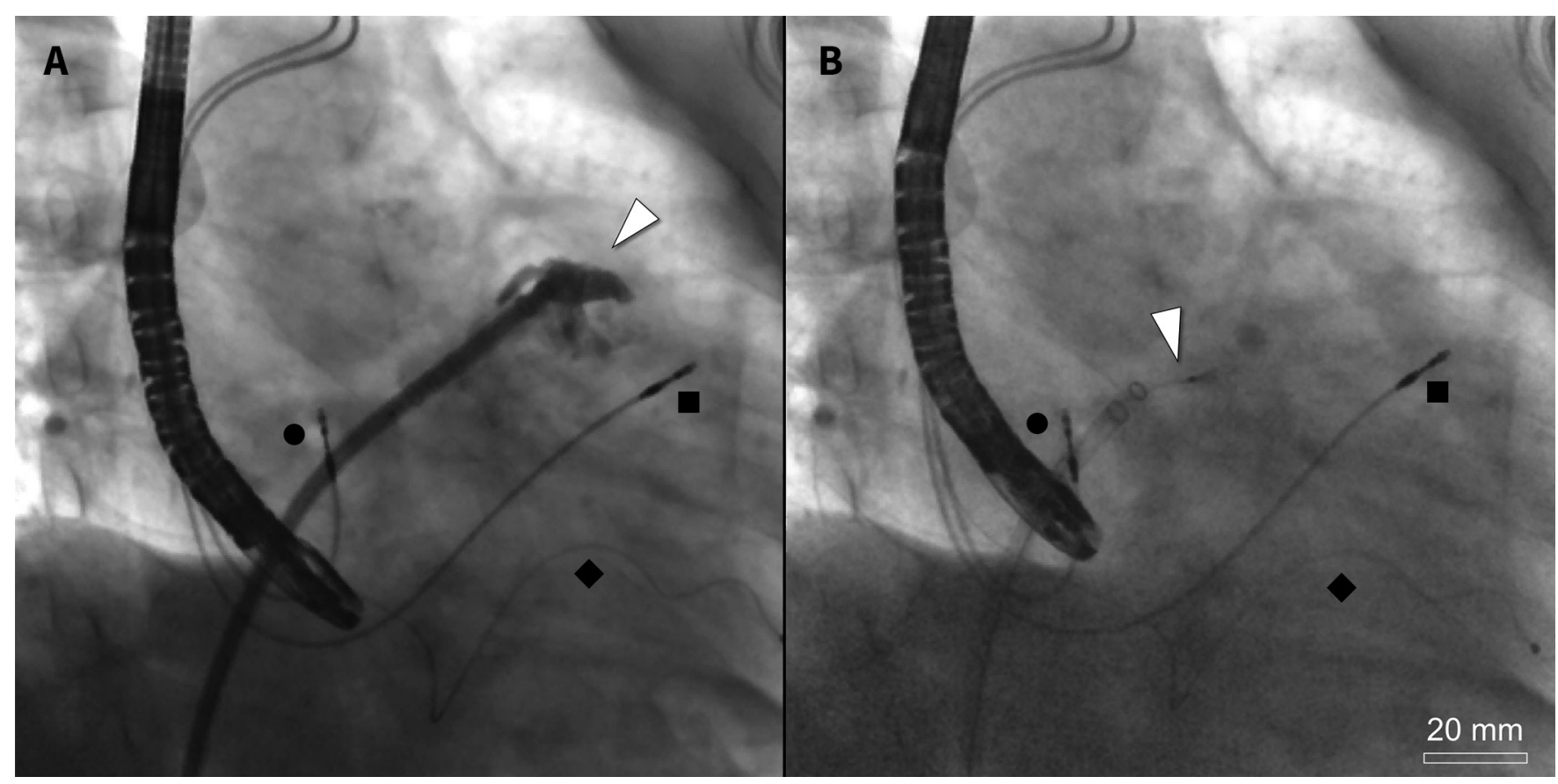

Figure 2: Deployment of a left atrial appendage closure (LAAC) device. (A) A delivery catheter is shown crossing the interatrial septum and directed toward the left atrial appendage (LAA), which is opacified with contrast (arrowhead). (B) LAAC device positioning in the LAA is confirmed before being released (arrowhead). A transesophageal echocardiographic probe is visible on the left. Leads from a cardiac resynchronization therapy device are seen in the right atrium $(\bullet)$, right ventricle $(\square)$ and coronary sinus $(\bullet)$

Feasibility Study With Watchman Left Atrial Appendage Closure Technology) study examined the safety of left atrial appendage closure in patients in whom oral anticoagulation had been deemed a contraindication. Patients underwent the closure procedure with the Watchman device, and received clopidogrel and ASA for six months, followed by ASA indefinitely. Among the 150 patients treated, the annualized event rate was $2.3 \%$ for all-cause stroke or systemic embolism, $0.6 \%$ for hemorrhagic stroke and $5.0 \%$ for nonprocedure- or device-related cardiovascular or unexplained death. The observed rate of ischemic stroke with the Watchman was $1.7 \%$ per year - 64\% lower than the expected rate (based on a $\mathrm{CHADS}_{2}$ score of 2.8 on antiplatelet therapy alone). ${ }^{6}$ Similar findings have been reported by Saw and colleagues in an early Canadian experience with the initial 106 patients implanted with the Watchman. ${ }^{9}$ The ASAP-TOO study (Assessment of the WATCHMAN ${ }^{\text {TM }}$ Device in Patients Unsuitable for Oral Anticoagulation; clinicaltrials.gov, no. NCT02928497) is likely to inform the role of left atrial appendage closure in patients who have contraindication to anticoagulation therapy.

Data on the Amulet device in these patients are of comparable quality to that of the Watchman device. In a multicentre, registrybased study involving 52 Canadian patients with atrial fibrillation at high risk of cardioembolic events (mean $\mathrm{CHADS}_{2}$ score of 3 ) and who had absolute contraindications to anticoagulant therapy, left atrial appendage closure with the Amulet device followed by dual- or single-antiplatelet therapy was associated with annualized rates of $1.9 \%$ for stroke, $0 \%$ for systemic embolism, $1.9 \%$ for major bleeding and $5.8 \%$ for death over a mean follow-up of 20 months. $^{7}$ The cumulative event rates were $87 \%$ (stroke), $66 \%$ (stroke, transient ischemic attack, systemic embolism) and 61\% (major bleeding) lower than those expected based on $\mathrm{CHADS}_{2}$, $\mathrm{CHA}_{2} \mathrm{DS}_{2}$-VASc and HAS-BLED scores, respectively. These findings have been replicated in European registries comparing the Amulet device to medical therapy with an antiplatelet regimen alone. ${ }^{11}$

\section{What are the harms?}

Complications from left atrial appendage closure include vascular access site bleeding, device embolization, pericardial effusion with and without tamponade, stroke and death. ${ }^{9}$ In the contemporary era, implantation success can be accomplished in $95 \%$ or more of patients. ${ }^{12}$ In a procedural performance study after FDA approval that involved 3000 consecutive patients, the rate of procedure- or device-related complications was $1.02 \%$ for pericardial tamponade, $0.29 \%$ for pericardial effusion, $0.08 \%$ for stroke, $0.24 \%$ for device embolization and $0.08 \%$ for death. About $66 \%$ of pericardial tamponade events (the most common major procedural complication) were treated percutaneously (i.e., without cardiac surgery). ${ }^{13}$ Most recently, in an analysis of all 1739 patients who received the Watchman implant in four prospective FDA studies, device-related thrombus was seen in 65 patients (3.74\%) and was associated with a higher rate of stroke (rate ratio $3.55,95 \%$ confidence interval $[\mathrm{Cl}] 2.18-5.79$ ) and systemic embolism (rate ratio $3.22,95 \% \mathrm{Cl} 1.90-5.45) .{ }^{14}$ Notably, although the risk of stroke or systemic embolization is higher in patients with device-related thrombus, most events occurred in patients without documented device-related thrombus. ${ }^{14}$ Finally, data from left atrial appendage closure trials now exceed five years of follow-up, but longer-term safety data of implants and unforeseen risks remain to be evaluated. 


\section{What can be expected in the future?}

Pending results from ongoing trials, left atrial appendage closure may offer an alternative for stroke prevention in selected patients with atrial fibrillation, particularly those with contraindications to oral anticoagulation and recurrent events despite optimal medical therapy. In particular, comparisons between specific left atrial appendage closure devices, the benefit and risk profiles of closure strategies in select risk cohorts of patients, and comparative data relative to direct oral anticoagulants are still needed.

\section{References}

1. Healey JS, Oldgren J, Ezekowitz M, et al.; RE-LY Atrial Fibrillation Registry and Cohort Study Investigators. Occurrence of death and stroke in patients in 47 countries 1 year after presenting with atrial fibrillation: a cohort study. Lancet 2016;388:1161-9.

2. Hsu JC, Maddox TM, Kennedy KF, et al. Oral anticoagulant therapy prescription in patients with atrial fibrillation across the spectrum of stroke risk: insights from the NCDR PINNACLE Registry. JAMA Cardiol 2016;1:55-62.

3. O'Brien EC, Holmes DN, Ansell JE, et al. Physician practices regarding contraindications to oral anticoagulation in atrial fibrillation: findings from the Outcomes Registry for Better Informed Treatment of Atrial Fibrillation (ORBIT-AF) registry. Am Heart J 2014;167:601-9.e1.

4. Blackshear JL, Odell JA. Appendage obliteration to reduce stroke in cardiac surgical patients with atrial fibrillation. Ann Thorac Surg 1996;61:755-9.

5. Reddy VY, Doshi SK, Kar S, et al. 5-year outcomes after left atrial appendage closure: from the PREVAIL and PROTECT AF trials. J Am Coll Cardiol 2017;70:2964-75.

6. Reddy VY, Möbius-Winkler S, Miller MA, et al. Left atrial appendage closure with the Watchman device in patients with a contraindication for oral anticoagulation: the ASAP study (ASA Plavix Feasibility Study With Watchman Left Atrial Appendage Closure Technology). J Am Coll Cardiol 2013;61:2551-6.

7. Urena M, Rodés-Cabau J, Freixa X, et al. Percutaneous left atrial appendage closure with the AMPLATZER cardiac plug device in patients with nonvalvular atrial fibrillation and contraindications to anticoagulation therapy. J Am Coll Cardiol 2013;62:96-102.

8. Holmes DR Jr, Doshi SK, Kar S, et al. Left atrial appendage closure as an alternative to warfarin for stroke prevention in atrial fibrillation: a patient-level metaanalysis. J Am Coll Cardiol 2015;65:2614-23.

9. Saw J, Fahmy P, Azzalini L, et al. Early Canadian multicenter experience with WATCHMAN for percutaneous left atrial appendage closure. J Cardiovasc Electrophysiol 2017;28:396-401.
10. Sahay S, Nombela-Franco L, Rodes-Cabau J, et al. Efficacy and safety of left atrial appendage closure versus medical treatment in atrial fibrillation: a network meta-analysis from randomized trials. Heart 2017;103:139-47.

11. Tzikas A, Shakir S, Gafoor S, et al. Left atrial appendage occlusion for stroke prevention in atrial fibrillation: multicentre experience with the AMPLATZER Cardiac Plug. Eurolntervention 2016;11:1170-9.

12. Boersma LV, Schmidt B, Betts TR, et al.; EWOLUTION investigators. Implant success and safety of left atrial appendage closure with the WATCHMAN device: periprocedural outcomes from the EWOLUTION registry. Eur Heart J 2016;37:2465-74.

13. Reddy VY, Gibson DN, Kar S, et al. Post-approval U.S. experience with left atria appendage closure for stroke prevention in atrial fibrillation. J Am Coll Cardiol 2017;69:253-61.

14. Dukkipati SR, Kar S, Holmes DR Jr, et al. Device-related thrombus after left atrial appendage closure: incidence, predictors, and outcomes. Circulation 2018 May 11 [Epub ahead of print]. doi: 10.1161/CIRCULATIONAHA.118.035090.

\section{Competing interests: None declared.}

This article has been peer reviewed.

The authors have obtained patient consent.

Affiliations: Clinical Epidemiology Program (Khan), Ottawa Hospital Research Institute; School of Epidemiology and Public Health (Khan, Ramirez), University of Ottawa; CAPITAL Research Group (Ramirez, Hibbert), Division of Cardiology, University of Ottawa Heart Institute, Ottawa, Ont.

Contributors: All of the authors contributed to the conception and design of the work, and the acquisition, analysis, and interpretation of data. All of the authors drafted the manuscript, revised it critically for important intellectual content, gave final approval of the version to be published and agreed to be accountable for all aspects of the work.

Correspondence to: Benjamin Hibbert, bhibbert@ottawaheart.ca

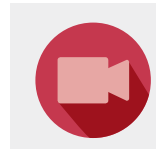

Three videos showing the left atrial appendage closure procedure are available online in Appendices 1-3 (at www. cmaj.ca/lookup/suppl/doi:10.1503/cmaj.180470/-/DC1). 